\title{
Historias que enseñan a estudiar y aprender: Una experiencia en la enseñanza obligatoria portuguesa
}

\section{Pedro Rosário ${ }^{1}$, José Carlos Núñez Pérez ${ }^{2}$ y Julio Antonio González-Pienda ${ }^{2}$}
${ }^{1}$ Departamento de Psicología. Universidad do Minho. Braga.
${ }^{2}$ Departamento de Psicología. Universidad de Oviedo.

\section{Portugal \\ prosario@iep.uminho.pt}

\section{Resumen}

En el marco de los nuevos planes de estudios de la educación básica portuguesa, hemos llevado a cabo un proyecto de investigación desarrollado en el Colégio de Nossa $\mathrm{Sr}^{\mathrm{a}}$ Rosário y coordinado por el primer autor de este artículo. La autorregulación del aprendizaje es el marco conceptual de este proyecto: “(Des)venturas do Testas". Basándonos en el marco teórico sociocognitivo, presentamos y analizamos el modelo de las fases cíclicas de la autorregulación del aprendizaje, la naturaleza de las estrategias de aprendizaje y algunas implicaciones para la práctica educativa. El carácter innovador de esta propuesta se relaciona con el uso de "historias" como un medio para transmitir y discutir las estrategias de estudio, planteadas por un modelo próximo. Pensamos que las aventuras y (des)venturas do Testas, un estudiante como cualquier otro, permitirán a los alumnos una identificación fácil con las propuestas de resolución discutidas, así como el traslado de las competencias discutidas en el aula, para otros dominios escolares y su propia vida.

Palabras Clave: Independent Study, self-regulated learning, learning strategies, narrative. 


\section{Introducción}

- Maestro, tú nos cuentas historias pero no nos explicas su significado...

- Déjame que te ofrezca una manzana. Para facilitarte la vida la pelaré yo - el alumno lo agradeció. - ¿Puedo cortarla en pequeños trozos?

- Me encantaría, pero...

- Permíteme también que mastique la manzana antes de que la comas...

- Maestro, eso no me gustaría - intervino el joven.

- Pues, explicarte cada historia sería como masticar yo tu manzana.

(Adaptado de la sabiduría tibetana)

El currículo de la enseñanza básica en Portugal, sinónimo de enseñanza obligatoria, comprende los cursos entre $1 .^{\circ}$ (correspondiente a 6 años de edad) hasta 9. (aproximadamente 14 años). En este curso 2003/4, terminará la extensión a toda la enseñanza básica del articulado propuesto en la última reorganización curricular iniciada en 2001. En el preámbulo de la Ley 6/2001 (ver Abrantes, 1999) que la organiza, podemos leer que su objetivo estratégico es "la garantía de una base de educación para todos, defendiendo como principio, un proceso de educación y formación a lo largo de la vida." En esta ley se sostiene que "se necesita promover un cambio gradual en las prácticas de administración curricular. Esta reorganización tiene como objetivo mejorar la respuesta educativa a los problemas típicos de la diversidad de los contextos escolares y asegurar que todos aprenden más y de una manera más significativa" (Abrantes, 1999, p.6). Una de las propuestas de este nuevo desarrollo curricular propone tres nuevas áreas curriculares no disciplinares donde se hace efectiva la articulación entre las competencias transversales que articulan el trabajo del plan de estudios nacional portugués. Estas tres nuevas áreas curriculares no disciplinares se denominan "Estudo Acompanhado" (Estudio Acompañado), “Área de Formação Cívica” (Área de Formación Cívica) y “Área de Projecto" (Área de Proyecto). Cada una de estas áreas novedosas presenta una curricular no opcional con 90 minutos por semana para los cursos de 5. ${ }^{\circ}$ a $9 .^{\circ}$. En el $1 .^{\circ}$ Ciclo do Ensino Básico Portugués (correspondiente a $1^{\circ}$ y $2^{\circ}$ Ciclos de Primaria en España), de $1 .^{\circ}$ a $4 .^{\circ}$ año (6 a 9 anos de edad), el trabajo en estas áreas curriculares no presenta un formato rígido en el horario de los estudiantes, lo cual no ocurre en los demás ciclos de la enseñanza. La evaluación del trabajo desarrollado en estas nuevas áreas, por su naturaleza promocional (de desarrollo) y no reeducativa, asume un formato cualitativo.

En este artículo nos concentraremos en los aspectos que conciernen al área curricular de "estudio acompañado". Así, en la Ley 6/2001 se declara que el "Estudio Acompañado" 
busca la adquisición de competencias que permitan a los estudiantes la apropiación de métodos de estudio y de trabajo y proporcionen el desarrollo de actitudes y capacidades que favorezcan el desarrollo de la autonomía en el logro de los aprendizajes" (Artículo 5. ${ }^{\circ}$, apunte 3, párrafo b).

El Proyecto (Des)venturas do Testas que discutiremos a continuación, se elaboró en Colegio de "Nossa Senhora do Rosario" - Oporto (Portugal) -, con un grupo de maestros y de psicólogos para aplicar en el Estudio Acompañado. Esta herramienta educativa supone una verdadera oportunidad para vincular la investigación académica y la práctica educativa. Como fruto de este intercambio nació este proyecto de desarrollo de competencias en el estudio, anclado en un marco teórico sólido, pero también en el conocimiento de la realidad de la escuela. Este equipo multidisciplinar, que incluye a maestros, psicólogos, directores e investigadores universitarios, con esta propuesta situada en el espacio del "Estudio Acompañado" pretende aportar a los estudiantes una oportunidad efectiva para aprender a aprender, mediante la promoción o desarrollo de las competencias de autorregulación.

Ante todo, este es un proyecto para promover el aprendizaje estratégico. La autorregulación del aprendizaje se refiere al grado en que los estudiantes se comprometen metacognitiva, motivacional y comportamentalmente en su propio proceso de aprendizaje (Rosário, 2002a; Schunk \& Zimmerman, 1994). Los alumnos pueden autorregular diferentes dimensiones del aprendizaje, por ejemplo, sus motivos para aprender, los métodos y estrategias de aprendizaje que utilizan, los resultados del aprendizaje y los recursos sociales y ambientales de su entorno (González-Pumariega, Núñez, Cabanach \& Valle, 2002). Se refiere, así, a aspectos cualitativos y cuantitativos del proceso de enseñanza-aprendizaje, incluyendo las estrategias que los estudiantes usan, la frecuencia y la habilidad en su utilización. Los pilares del proceso de autorregulación son la opción y el control (Schunk \& Zimmerman, 1994, 1996). Y "la tarea principal de los maestros es conseguir que los estudiantes se impliquen en las actividades de aprendizaje para lograr alcanzar los resultados pretendidos... asumiendo que lo que los estudiantes logran es más importante para la determinación del aprendizaje que lo que el maestro hace" (Shuell, 1986, p.429).

Anclados en este marco teórico, defendemos que la promoción de competencias autoregulatórias para desarrollar en el aula no debe confinarse a la manipulación de un conjunto de estrategias de aprendizaje aisladas y descontextualizadas, sino a la discusión de una lógica 
que prepare, guiando todo el trabajo de los estudiantes, fortaleciendola cualitativamente. Los estudiantes no pueden autorregular su aprendizaje a menos que le sean proporcionadas oportunidades, y ellos puedan controlar algunas dimensiones esenciales de su aprender. La importancia de la autorregulación del aprendizaje es subrayada por la investigación en el área que sugiere, recurrentemente, que los alumnos que autorregulan su aprendizaje están mentalmente activos durante el aprendizaje ejerciendo un control sobre el establecimiento y la prosecución de los objetivos establecidos (Weinstein, 1994; Zimmerman, 1998, 2000). El proyecto de promoción del aprendizaje autorregulado que nosotros proponemos, se basa en la convicción de que todos los estudiantes son capaces aprender y autorregular la motivación y el aprendizaje, siempre que decidan aprender asumiendo el control de las tareas de aprendizaje.

Sin embargo, muchos estudiantes de nuestras escuelas presentan disfunciones en su procesamiento autorregulatorio, en el dominio motivacional (por ejemplo, evitando determinadas tareas o rindiéndose antes de la conclusión), en los métodos que usan (por ejemplo, el uso inadecuado de estrategias de aprendizaje) o en los recursos usados (por ejemplo, no buscar la ayuda de padres o compañeros cuando lo necesitan). Este tipo de problemas, suele surgir, cuando los estudiantes no conocen los procesos de autorregulación del aprendizaje o utilizan los métodos de "siempre" para enfrentarse a las tareas escolares. Por otra parte, las escuelas tampoco proporcionan el entrenamiento autorregulatório necesario a sus maestros y estudiantes, por falta de tiempo, espacio, formación docente... (Rosário, 2001a, 2002a, 2003).

El programa de intervención en el área del desarrollo de competencias de autorregulación del proceso de aprendizaje que ahora presentamos -materializado en un colección intitulada “(Des)venturas do Testas" -, está constituido por un libro de narraciones con historias, en las que se hace explícito el proceso de autorregulación, para trabajar en los cursos de la enseñanza obligatoria portuguesa $\left(5 .^{\circ}, 6 .^{\circ}, 7 .^{\circ}, 8 .^{\circ}\right.$ y 9. ${ }^{\circ}$ curso). En este programa, Testas, el héroe, cuenta a sus amigos algunos de sus éxitos, fracasos, aventuras y desventuras en su proceso de estudio y aprendizaje (Rosário, 2002b,c,d). Este proyecto esta inserto en un marco de innovación educativa participada (Randi \& Corno, 2000; Rosário, 2002a), que caracterizamos como un proceso en el cual maestros y investigadores trabajan conjuntamente, aprovechando las sinergias de la conexión teoría-práctica para la construcción de herramientas instruccionales.

Las “(Des)venturas do Testas" no intenta ser "un proyecto más”, concebido en un despacho fuera de toda realidad de las aulas, y por eso, desfasado de las necesidades reales de la 
intervención educativa. Este programa es el producto de una (co)construcción en el ejercicio de la innovación educativa, asumiendo, por esta razón, un marco ecológico bien definido. Esta herramienta curricular se apoya en la convicción de que el aprendizaje autorregulado puede promoverse a través de la modelación y experienciación de oportunidades múltiples para el desarrollo de un aprendizaje independiente (Pintrich, 2000; Rosário, 2003). Nuestro principal objetivo ha sido crear, en el espacio-escuela, una atmósfera de aprendizaje que proporcione la implicación de los padres, maestros y estudiantes en la construcción de un guión de aprendizaje autorregulado. Hemos intentado evitar la descontextualización de las experiencias concretas del aprendizaje, severamente sancionada en la literatura como una metodología que no sólo no promueve el traslado de conocimiento y competencias para otros entornos educativos, sino que además fomenta entre los estudiantes la creencia de que las estrategias de aprendizaje son un kit de tácticas dispuestas para usar indiferenciadamente de las diversas tareas de aprendizaje.

Las “(des)venturas do Testas" entrelazan historias en un discurso intimista y narrativo dónde un estudiante cualquiera, describe y reflexiona sobre sus experiencias concretas de aprendizaje. Sus compañeros-lectores pueden, de esta manera, experimentar un aprendizaje vicario a través de esta narración y aprender inductivamente un modelo autorregulado para poder afrontar sus experiencias de aprendizaje. En los distintos textos de las diferentes (des)venturas, las estrategias de aprendizaje están diseminadas conforme el modelo de autorregulación propuesto por el marco sociocognitivo (Zimmerman \& Martínez-Pons, 1986, 1988).

Nuestra propuesta de trabajo con, y para, los estudiantes sugiere la tarea inversa: identificar las estrategias de aprendizaje subyacentes al discurso, construyendo la propia historia de autorregulación, a partir de la narración ofrecida por el Testas. La historia que nosotros ofrecimos no es, por consiguiente, un producto final "redondo". Al contrario, surge como el punto de partida para la construcción personal de itinerarios autorregulación, por parte de los padres, de los maestros y de los estudiantes.

De acuerdo con Bruner (1990), estas narraciones no buscan hacer a los alumnos mejores lectores, sino "mejores autores". No obstante, la propuesta no es, obviamente, disyuntiva. Para lograr que los alumnos crezcan en la capacidad de autorregulación, tenemos que incrementar sus competencias lectoras y reflexivas. Por este motivo, en este programa de interven- 
ción nos proponemos usar las historias de "Testas" como una ventana a través de la cual se puedan "construir" momentos de autorregulación.

Las narraciones siguen un guión consonante con el marco teórico sociocognitivo (Pintrich, 2000; Schunk, 1996; Schunk \& Zimmerman, 1998; Zimmerman, 1998, 2000). En éstas, algunos aspectos ilustran esta intención de invitar al estudiante a la construcción de este proyecto personal. Por ejemplo, el carácter flexible de las sesiones, sin tiempos rígidos ni actividades normalizadas, lleva a la construcción de un "guión personal” autorregulado (e.g., Rosário, 2001b, 2002a). En las claves de trabajo presentadas en el libro teórico que sostiene el proyecto (Rosário, 2002; 2003), se propone un conjunto amplio de sugerencias para trabajar las competencias de autorregulación, dejando a los educadores la opción de las actividades apropiadas a cada alumno/grupo-classe.

\section{Modelo de autorregulación}

Barry Zimmerman y colaboradores presentaron en 1998, y reiterandolo en 2000, un modelo explicativo del aprendizaje autorregulado. La intervención que estamos describiendo, aunque anclado en el marco sociocognitivo (Zimmerman, 1998, 2000), presenta a un modelo cíclico más parsimonioso: PLE- Planificación, Ejecución y Evaluación de la tarea (Rosário, 2002a). Como puede observarse en la ilustración 1, el modelo presenta tres fases: Planificación, Ejecución y Evaluación de las tareas desarrolladas.

La fase de la planificación tiene lugar cuando los estudiantes analizan la tarea específica de aprendizaje. Este análisis implica el estudio de los propios recursos personales y del entorno para afrontar la tarea concreta, el establecimiento de objetivos específicos y un plan para alcanzarlos. La fase de ejecución se refiere a la aplicación de la estrategia para alcanzar la meta. Los estudiantes utilizan un conjunto organizado de estrategias de aprendizaje y monitorizan su eficacia para alcanzar la meta establecida. Por último, la fase de la evaluación tiene lugar cuando el estudiante analiza la relación entre el producto de su aprendizaje y la meta establecida previamente. El núcleo fundamental de esta fase del proceso de autorregulación no se centra en la comprobación, sin más, de las diferencias entre el producto de aprendizaje y la meta, sino también en la reflexión sobre qué estrategias podrían utilizarse para reducir esa distancia. 
desarrollo y la discusión de temas relacionados con cada una de las fases, todos los capítulos se organizan para discutir el proceso autorregulación como un todo. Estas dos lógicas deben ser respetadas y asumidas por los educadores que trabajen con este instrumento para la promoción del aprendizaje autorregulado, garantizando su natureza y coherencia (Rosário, 2002a).

\section{Arquitectura del proyecto "(Des)venturas do Testas"}

La intervención que nosotros proponemos no se configura como un programa de competencias del estudio. No propone una estructura de sesiones distribuida a lo largo del curso, ni prevé tiempos determinados y justos para desarrollar actividades previamente estipuladas. Al contrario, el proyecto (Des)venturas do Testas es una herramienta para trabajar las competencias autorreguladoras en el aula, que a pesar de poseer un guión sólido que organiza el proyecto (Rosário, 2002), presenta un carácter ajustable a las diferentes velocidades y necesidades de los lectores-autores. La autorregulación del aprendizaje es un proceso intencional: los estudiantes deben aprender a usar estrategias de aprendizaje intencional y autónomamente en su trabajo personal. Algunas estrategias se encuentran dirigidas a organizar el procesamiento de la información, mientras otras ayudan a la gestión del tiempo o las emociones... (Cabanach, Valle, Rodríguez \& Piñeiro, 2002; Rodríguez, Cabanach, \& Piñeiro, 2002; Rosário, 2001a,b, 2002, 2003; Schunk, 1998; Zimmerman, 1998, 2000). Algunas estrategias son específicas de la tarea mientras otras son más generales. Sin embargo, a pesar de la variedad de las estrategias de aprendizaje, éstas comparten un conjunto de características. En primer lugar, las estrategias constituyen acciones deliberados para alcanzar objetivos específicos. En segundo, se corresponden con una respuesta personal a un problema dado, proceso que conlleva implicación y control personal en las tareas a realizar, más que el uso de un guión estratégico, predefinido, para afrontar las tareas de aprendizaje. En tercer lugar, las estrategias son selectiva y flexiblemente aplicadas a las tareas, implicando tanto recursos cognitivos como motivacionales. Por último, las estrategias de aprendizaje deben entrenarse en diferentes tipos de tareas escolares para facilitar su transferencia. Como ya indicamos, en el modelo sociocognitivo, la autorregulación no es contemplada como un rasgo psicológico, sino como una competencia en relación muy estrecha con un contexto específico (Schunk \& Zimmerman, 1994). Lo que es corroborado por el hecho de que los aprendices no se implican en comportamientos autorreguladores de una manera similar en todos los contextos y tareas de aprendizaje. A pesar de la transversalidad de algunos procesos autorregulatorios - por ejemplo, el establecimiento de los objetivos -, los estudiantes deben aprender a adaptar, con eficacia, sus procesos 
de aprendizaje a los distintos dominios de aprendizaje. Recordamos que los ejes del proceso autorregulatório son la opción y el control.

Esta metodología que nos proponemos trabajar en la asignatura de "Estudo Acompanhado", pero también en casa, constituye una innovación tanto para los estudiantes como para los educadores. Por esta razón, es importante informar a los padres y a los profesores sobre la tipología de estrategias de aprendizaje sugeridas en las “(Des)venturas do Testas” y promover, así, su desarrollo en el trabajo.

La explicación del profesor/educador, la discusión y el logro de las actividades en la escuela o en casa, debe seguir el ritmo y la dirección de las reflexiones logradas por los estudiantes. Sugerimos la elaboración de registros y sumarios de los aspectos principales discutidos en las sesiones de trabajo para que, después, los estudiantes puedan reflexionar sobre los aprendizajes. Los cuadernos de la colección “(Des)venturas do Testas” pueden leerse y discutirse, en la escuela y casa, respetando la lógica del proceso de autorregulación del aprendizaje ya descrito: cada actividad/tarea/problema/história deben planearse, ejecutarse y evaluarse (Rosário, 2002a, b, c, d). La asimilación de estos tres pasos ayudará a los estudiantes y a los educadores en la reflexión sobre su participación en el proceso y a repensar sus opciones estratégicas hacia las distintas tareas de aprendizaje. La promoción de las competencias autorregulatorias es crucial para el crecimiento de los estudiantes. Si éstos conocen qué estrategias de aprendizaje utilizar en su estudio, cómo y cuándo, este repertorio conductual tendrá consecuencias en la calidad de su aprendizaje y bienestar personal, además de incrementarse sus niveles de autoeficacia académica y su autoestima (Valle, Cabanach, Núñez, GonzálezPienda, Rodríguez \& Piñeiro, 2003).

\section{Conclusión}

Las estrategias de aprendizaje necesitan ser aprendidas y practicadas dentro de un contexto siguiendo una rutina "más exigente" que la administración de fichas aisladas. El enfoque de intervención no debe centrarse en la compensación de un déficit, en el presupuesto que el trabajo de los estudiantes se basa en estrategias de aprendizaje inadecuadas, necesitando, por consiguiente, de intervención "reeducativa". Al contrario, los datos referenciados en las diferentes revisiones de la literatura en este área de conocimiento (Simpson et al., 1997) sugieren que la enseñanza y práctica de las estrategias de autorregulación del aprendizaje deben seguir una lógica que implica al alumno en la secuencia "Escoger- controlar - reflexionar". 
Los estudiantes necesitan conocer, lo más ampliamente posible, estrategias de autorregulación del aprendizaje, conocer su aplicación a las distintas situaciones y tareas de aprendizaje, pero también cuando aplicar cada una. La enseñanza y práctica de las estrategias de aprendizaje no debería ser enfocada como una tarea cerrada en un curso concreto o área curricular específica, sino como una responsabilidad de todos los maestros de ese grupo de alumnos. La ausencia de referencia y eco del trabajo estratégico en los distintos contextos de aprendizaje, compromete la efectividad de los aprendizajes realizados y su transferencia a otros dominios. El perfil de trabajo que proponemos para el "Estudo Acompanhado" obedece a nuestro convencimiento de que es necesario y urgente formar a los maestros en las estrategias instruccionales válidas para elicitar y afianzar en los estudiantes la habilidad para autorregular su propio proceso de aprendizaje. Y ello no sólo desde una perspectiva reeducativa o compensadora de déficits, con acciones de formación puntuales, sino también desde una vertiente preventiva, por ejemplo, incluyendo este tema en la formación inicial de los maestros o en su formación posterior. Otro de los aspectos estructurantes de este área curricular no disciplinar está relacionado con el desarrollo de una articulación con todas las partes implicadas en la educación. Esta sinergia podría ser potencializada, entre otras alternativas, a través de las sugerencias de tareas escolares para desarrollar en los espacios extra-escolares.

En esta línea, somos críticos con la enseñanza de estrategias de aprendizaje "separadas" del plan de estudios, aunque reconocemos su utilidad en casos puntuales de estudiantes con dificultades de aprendizaje. Por otra parte, defendemos que el desarrollo de las competencias en autorregulación de los estudiantes es dependiente de las actividades concretas de aprendizaje desarrolladas en los contextos específicos del aprendizaje. Este formato curricular del "Estudo Acompanhado" puede ser el primer paso para demostrar cómo puede ser una enseñanza de estrategias de autorregulación integradas curricularmente.

Por tanto, ¿qué aprendemos cuándo aprendemos? Recordemos una vieja historia que resume de una manera muy gráfica el desafío educativo que todos los educadores tenemos: enseñar a nuestros estudiantes a pensar. Había un estudiante que nunca consiguió convertirse en un buen matemático porque "creía ciegamente" en las respuestas de las últimas páginas de su manual y, aunque pueda parecer paradójico, las respuestas eran las correctas. Esperamos que las “(Des)venturas do Testas" puedan constituirse como una oportunidad para promover la autorregulación en el aula y en casa, implicando a estudiantes, maestros y padres. 


\section{Referencias}

Abrantes, P. (1999). Gestão flexível do currículo. Lisboa: Ministério da Educação. Departamento da Educação Básica.

Bruner, J. (1990). Acts of meaning. Cambridge, MA: Harvard University Press.

Cabanach, R.G., Valle, A., Rodríguez, S. \& Piñeiro, I. (2002) Autorregulación del aprendizaje y estrategias de estudio. En J.A. González-Pienda, R.G. Cabanach, J.C. Núñez \& Valle (coord.), Manual de Psicología de la Educación,17-38. Madrid:Pirámide.

González-Pumariega, S., Núñez, J.C., Cabanach, R.G. \& Valle, A. (2002). El aprendizaje escolar desde una perspectiva psicoeducativa. En J.A. González-Pienda, R.G. Cabanach, J.C. Núñez \& Valle (coord.), Manual de Psicología de la Educación, 41-68. Madrid:Pirámide.

Pintrich, P. R. (2000). An achievement goal theory perspective on issues in motivation terminology, theory and research. Contemporary Educational Psychology, 25, 92-104.

Randi, J., \& Corno, L. (2000). Teacher innovations in self-regulated learning. In M. Boekaerts, P. Pintrich \& M. Zeidner (Eds.), Handbook of Self-regulation, 651-689. New York: Academic Press.

Rodríguez, S., Cabanach, R.G., \& Piñeiro (2002). Gestión de recursos y estrategias motivacionales. En J.A. González-Pienda, R.G. Cabanach, J.C. Núñez \& Valle (coord.), Manual de Psicología de la Educación, 145-164. Madrid:Pirámide.

Rosário, P. (2001a) Diferenças processuais na aprendizagem: Avaliação alternativa das estratégias de auto-regulação da aprendizagem. Psicologia, Educação e Cultura, 1(1), 87-102,

Rosário, P. (2001b). Área curricular de "Estudo Acompanhado". Contributos para a discussão de uma metodologia. Revista Portuguesa de Educação, 14 (2), 63-93.

Rosário, P. (2002a). Estórias para estudar, história sobre o estudar: Narrativas autoregulatórias na sala de aula. Porto: Porto Editora.

Rosário, P. (2002b). Testas para sempre. Porto: Porto Editora.

Rosário, P. (2002c ). Elementar, meu caro Testas. Porto: Porto Editora.

Rosário, P. (2002d). 007. ${ }^{\circ}$ Ordem para Estudar. Porto: Porto Editora.

Rosário, P. (2003). O Senhor aos papéis, a Irmandade do Granel. Porto: Porto Editora.

Schunk, D. H., \& Zimmerman, B. J. (1994). Self regulation in education: Retrospect and prospect. In D. H. Schunk \& B. J. Zimmerman (Eds.). Self-regulation of learning 
and performance: Issues and educational applications, 305-314. Hillsdale, NJ: Lawrence Erlbaum Associates, Inc.

Schunk, D. H., \& Zimmerman, B. J. (1996). Modeling and self-efficacy influences on children's development of self-regulation. In K. Wentzel \& J. Juvonen (Eds.), Social motivation: Understanding children's school adjustment, 154-180. New York: Cambridge University Press.

Schunk, D. H. (1996). Goal and self-evaluative influences during children's cognitive skill learning. American Educational Research Journal, 33, 359-382.

Schunk, D. H. (1998). Teaching elementary students to self-regulate practice of Mathematical skills with modeling. In D. H. Schunk \& B. J. Zimmerman (Eds.), Self-Regulated learning. From teaching to self-reflective practice, 137-159. Hillsdale, NJ: Lawrence Erlbaum Associates, Inc.

Schunk, D. H., \& Zimmerman, B. J. (1998). Conclusions and future directions for academic interventions. In D. H. Schunk \& B. J. Zimmerman (Eds.), Self-Regulated learning. From teaching to self-reflective practice, 225-234. Hillsdale, NJ: Lawrence Erlbaum Associates, Inc.

Shuell, T.J. (1986). Cognitive conceptions of learning. Review of Educational Research, 56, 411-436.

Simpson, M., Hynd, C., Nist, C., \& Burrel, K. (1997). College academic assistance programs and pratices. Educational Psychology Review, 9, 39-87.

Valle, A., Cabanach, R.G., Núñez, J.C., González-Pienda, J.A., Rodríguez, S. \& Piñeiro, I. (2003). Cognitive, motivational, and volitional dimensions of learning: A empirical test of hypothetical model. Research in Higher Education, 44, 557-580.

Weinstein, C. E. (1994). Strategic learning/strategic teaching: Flip sides of a coin. In P. R. Pintrich, D. R. Brown, \& C. E. Weinstein (Eds.), Student motivation, cognition, and learning: Essays in honor of Wilbert J. McKeachie, 257-273. Hillsdale, NJ: Erlbaum.

Zimmerman, B. J. (1998). Developing self-fulfilling cycles of academic regulation: An analysis of exemplary instructional models. In D. H. Schunk \& B. J. Zimmerman (Eds.), Self-Regulated learning. From teaching to Self-Reflective Practice, 1-19. Hillsdale, NJ: Lawrence Erlbaum Associates, Inc.

Zimmerman, B. J. (2000). Attaining self-regulation: A social cognitive perspective. In M. Boekaerts, P. Pintrich \& M. Zeidner (Eds.), Handbook of Self-regulation, 13-39. New York: Academic Press. 
Zimmerman, B. J., \& Martinez-Pons, M. (1986). Development of a structured interview for assessing student use of self-regulated learning strategies. American Educational Research Journal, 23 (4), 614-628.

Zimmerman, B. J., \& Martinez-Pons, M. (1988). Construct validation of a strategy model of student self-regulated learning. Journal of Educational Psychology, 80 (3), 284290. 
Historias que enseñan a estudiar y aprender: Una experiencia en la enseñanza obligatoria portuguesa.

[Página dejada en blanco intencionamente] 